\title{
Versuch der numerischen Erfassung des Reliefcharakters
}

Ludwig Ellenberg

\section{Einleitung}

Als Grundlage für morphologische Arbeiten ist es nötig zu wissen, wie groß die «Möglichkeit der Umgestaltung einer Landschaft» ist. Unter den mannigfaltigen Faktoren, die die Potenz der Landschaftsveränderung beeinflussen, ist der Reliefcharakter sehr wichtig. Er sollte deshalb numerisch erfaßt werden können, um eine Vergleichsbasis für verschiedene Gebiete zu liefern und die subjektive Beurteilung möglichst auszuschließen.

Schon früh wurde versucht, den Charakter des Reliefs zahlenmäßig auszudrücken. Dabei ging man verschiedene Wege; man ermittelte den Böschungswinkel, das Volumen über einer Basisfläche, die Flußdichte, die wahre Oberfläche oder die «Reliefenergie». Von Neuenschwander werden diese Methoden klar skizziert und kritisch betrachtet. Deshalb kann hier auf eine Übersicht verzichtet werden.

Vorgehen, mit denen man die Hangneigung bestimmt oder systematisch gelegte Profile auswertet, erfassen den Charakter des Reliefs als Ganzes ungenügend und greifen nur interessante Details heraus. Gutersohn, Krebs, Partsch, Schläpfer u. a. benützen die Reliefenergie, d. h. den relativen Höhenunterschied eines Gebietes, das als quadratische Einheitsfläche von 1, 4, $25 \mathrm{~km}^{2}$, und größer oder als "morphologisch einheitlicher Raum» definiert wird. Die Resultate bleiben oft unbefriedigend, da selbst bei überlegter Auswahl der Bezugsflächen nur eine Aussage über die Höhendifferenz zwischen höchstem und tiefstem Punkt erreicht wird. Auf welche Weise der Höhenunterschied überwunden wird, kann mit der Reliefenergie allein nicht ausgedrückt werden. (Bei gleicher Höhendifferenz sind in einem Falle die Hänge glatt und der Höhenlinienverlauf einfach; in einem anderen gliedern Taleinschnitte, Buckel und Furchen den Hang. Die «Reliefenergie» ist in beiden Fällen gleich groß, nicht aber der Charakter des Reliefs.) Die Reliefenergie müßte deshalb durch eine Größe ergänzt werden, die ausdrückt, auf welche Weise der Höhenunterschied überwunden wird, und die dem Relief des ganzen Bezugsgebietes Rechnung trägt.

Hiermit sei der Vorschlag gemacht, den Charakter des Reliefs durch zwei Größen zu kennzeichnen, die man zahlenmäßig fassen kann:

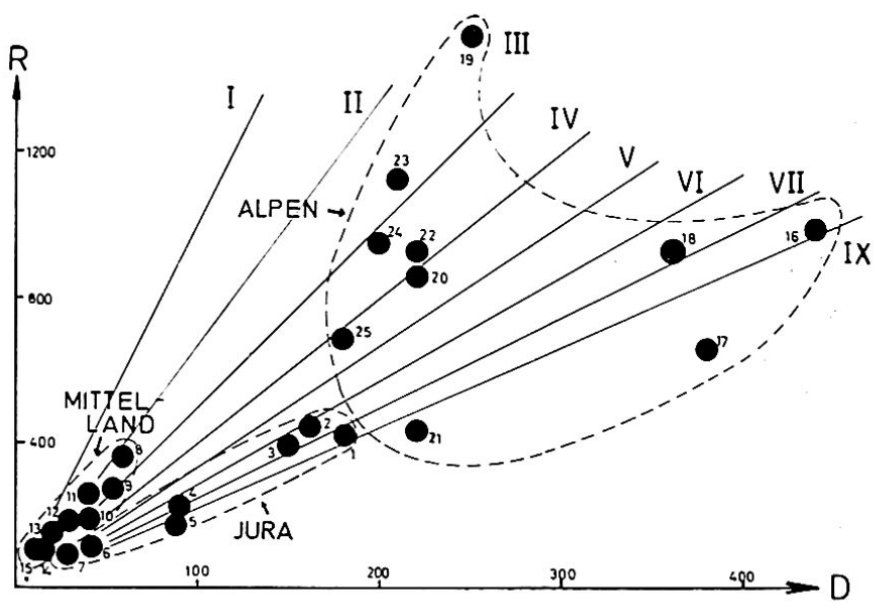

- Höhenkurvendichte D

- Reliefenergie R

\section{Die Höhenkurvendichte D}

Die Höhenkurvendichte, d.h. die Länge der Höhenkurven bestimmter Äquidistanz in der Einheitsfläche, wird in gleichmaßstäbigen Karten ermittelt, weil bei ihnen die Äquidistanz und die Generalisierung, die Verkürzung und Vereinfachung der wahren Höhenlinien im Kartenbild gleich sind. Im Falle der Schweiz empfiehlt sich die Landeskarte 1:50000 mit der Äquidistanz $20 \mathrm{~m}$. Da Vergleichszahlen und keine absoluten Werte gesucht werden, ist es unnötig, die wahre Länge der Höhenkurven zu bestimmen.

Die Einheitsfläche soll immer gleich groß sein. Sie darf nicht zu klein gewählt werden und zum Beispiel nur einen Hang konstanter Neigung erfassen, weil eine «morphologische Einheit» besser durch Form, Größe, Neigung charakterisiert werden kann. Sie darf aber auch nicht zu groß sein, da sonst innerhalb der Fläche der Reliefcharakter eine entscheidende Veränderung erfahren kann und $\mathrm{D}$ einen sinnlosen Mittelwert darstellt. Es empfiehlt sich eine Testfläche von $2 \times 2 \mathrm{~km}$, wie sie Gutersohn für die Ermittlung der Reliefenergie in den Alpen vorschlug. Schränkt man nicht auf gleichmaßstäbige Karten und fixierte Basisfläche ein, so läßt sich eine entsprechende Höhenkurvendichte (D') wie folgt berechnen: 


$$
\mathrm{D}^{\prime}=\frac{\text { Länge der Isohypsen in cm x Maßstabszahl M x Äquidistanz }}{\text { Bezugsfläche in } \mathrm{km}^{2}}
$$

Die Höhenkurvendichte, die Länge der Höhenkurven pro Einheitsfläche, ist um so größer,

je stärkere Höhenunterschiede bestehen, je größer die Hangneigung und je zerfurchter das Relief ist.

\section{Das Verhältnis der Reliefenergie zur Höhen- kurvendichte}

Bei gleichem $\mathrm{R}$ kann $\mathrm{D}$ ganz verschiedenen Wert haben. Ist D klein, so ist das Relief «einfach», das heißt wenig gegliedert. Ist $D$ groß, so sind die Hänge zerfurcht, der Höhenlinienverlauf kompliziert. Aus dem Verhältnis R:D läßt sich die Stärke der Zerfurchung ablesen, wie aus Abbildung 1 hervorgeht. Mit römischen Zahlen sind die Sektoren zunehmender Zerfurchung numeriert.

Für einige Testflächen im Jura, Mittelland und Alpen (J, M, A) wurden diese Werte berechnet. Im Mittelland Hänge an den Seen, Molasseberge, Schotterflächen mit ihren Randhängen usw., im Jura gewelltes Gelände der Freiberge, Hänge der eingeschnittenen Flüsse, Klusen, Kämme mit ihren Hängen usw. und in den Alpen zerfurchte Gipfelregionen, Karmulden, zerfurchte und glatte $\mathrm{Ab}$ stürze. In Tabelle 1 sind die Testflächen zusammengestellt und deren numerische Werte in Abbildung 1 veranschaulicht.

\section{Tabelle 1: Verwendete Testflächen}

\section{Nr. Koord.}

$1 \mathrm{~J} \mathrm{237/584} \mathrm{Klus} \mathrm{von} \mathrm{Pichoux.} \mathrm{Hänge.} \mathrm{Schmaler}$ Talboden.

2 J 243/578 Châtillon. Abhänge beidseits des Doubs.

3 J 235/563 Goumois. Abhänge beidseits des Doubs.

4 J 286/683 Langtal. Randenabfall bei Siblingen in Doggerkalk und Molasse.

5 J 287/686 Hemmental. Hänge und Talboden im Randen.

6 J 234/577 Les Genevez. Gewellte Landschaft der Freiberge.

7 J 232/570 Etang de la Gruère. Gewellte Landschaft der Freiberge.

8 M 243/681 Unterleimbach. Ostabhang des Uetlibergs und Hang des Aentlibergs.

9 M 233/689 Arn. Hang am Zürichsee südlich Käpfnach.
10 M 237/661 Schongau. Ostabhang des Hallwilersees.

11 M 239/689 Herrliberg. Hang zum Zürichsee.

12 M 253/677 Gubrist. Zum Teil moränenbedeckter Molassehügel, zwischen OberEngstringen und Regensdorf.

13 M 241/657 Boniswil. Sanft ansteigender Hang am Hallwilersee.

14 M 282/676 Klettgau. Schotterfläche zwischen Wilchingen und Trasadingen.

15 M 253/677 Ottenbach. Talboden der Reuss und sanft ansteigender Hang.

16 A 113/644 Fletschhorn. Gipfel- und Firnregion. Stark zerfurcht.

17 A 115/687 Valle Onserone. Südhang des Tals. Wild zerfurcht.

18 A 121/687 Valle di Vergeletto. Hänge beidseits im mittleren Teil.

19 A 113/639 Rothorn. SW-Abhang gegen Saas. Extreme Reliefenergie.

20 A 115/628 St. Niklaus. Linker Abhang des Mattertals im mittleren Teil.

21 A 152/687 Motta del Toro. Stark zerfurchter rechter Hang des Bedrettotals.

22 A 131/681 Valle di Bosco. Hang mit Talboden nördlich Bosco Gurin.

23 A 154/668 Vordere Grimsel. Steiler "Galen» zwischen Oberwald und Obergesteln.

24 A 119/622 Turtmanntal. Einfach gegliederter steiler Hang.

25 A 120/626 Oberes Ginals, Karmulde mit Hängen.

In Abbildung 1 gruppieren sich die Testflächen der drei Großlandschaften in einem jeweils typischen Bereich. Dabei wird der reiche Formenschatz der Alpen durch eine große Fläche dargestellt, der kleinere des Mittellandes und des Juras durch eine enger gefaßte. Es wird deutlich, daß in Alpenlandschaften die Höhenkurvendichte wechselnd, aber immer groß ist, was zum Teil von der ausgeprägten Reliefenergie und zum Teil von der starken Zerfurchung herrührt. Im Jura ist die Höhenkurvendichte ebenfalls sehr schwankend, aber kleiner als in den Alpen. Die Reliefenergie wechselt, bleibt aber gering wie im Mittelland.

Eine kartographische Darstellung der Ergebnisse ist auf zweierlei Weise möglich. Man kann D als Fläche darstellen (Helligkeitsabstufung oder Farbvariation) und $\mathbf{R}$ als Raster darüber abbilden. Besser wäre jedoch eine Darstellung einzelner Landschaftstypen, die sich aus der Lage der Punkte im Koordinatennetz der Abbildung ergeben. Dabei ist neben Reliefenergie und Höhenkurvendichte der 
"Zerfurchungsindex» (R.:D) zu beachten. So sind bei den Testgebieten 17 und 19 (Valle Onsernone und Rothorn) R und D wohl stark verschieden, besonders wichtig ist aber ihr gegenseitiges Verhältnis, welches durch den "Zerfurchungsindex» dargestellt wird. Sie stellen zwei vollkommen gegensätzliche Typen dar und liegen in verschiedenen «Zerfurchungssektoren" (Rothorn in Sektor III, Onsernone in Sektor IX). Ubbergangstypen lassen sich durch sinnvolles Aufgliedern der Umgrenzungsfläche ermitteln.

Bei diesen Úberlegungen bleibt zu prüfen, ob die numerischen Werte eine klarere Aussage beim Vergleich von Reliefcharakteren erlauben als die unmittelbare Anschauung aus der Karte.

\section{Literatur}

Brüning, K.: Die Reliefenergie des Harzes. Jb. d. Geogr. Ges. zu Hannover, 1927.

Flückiger, O.: Morphologische Untersuchungen am Napf. Habilitationsschrift, Univ. Zürich, 1919.

Finsterwalder, S.: Über den mittleren Böschungs- winkel und das wahre Areal einer topographischen Fläche.

Sitzungsberichte d. k. Bayerischen Akademie der Wiss., math.-phys. Klasse, Bd. 20, München 1890. Gutersohn, H.: Relief und Flußdichte. Dissertation Univ. Zürich, 1932.

Krebs, N.: Eine Karte der Reliefenergie Süddtlds. Pet. Mitt. 1922, Bd. 68.

Krebs, N.: Maß und Zahl in der physischen Geogr. Pet. Mitt. 1930, Erg. H. 209.

Neuenschwander, G.: Morphometrische Begriffe.

Dissertation Univ. Zürich, 1944.

Paschinger, V.: Die relativen Höhen von Kärnten. Pet. Mitt. 1934, Bd. 80.

Penck, A.: Morphologie der Erdoberfläche, Bd. 1, Stuttgart 1894.

Schläpfer, A.: Die Berechnung der Reliefenergie und ihre Bedeutung als graphische Darst. Dissertation Univ. Zürich 1938.

Smith, G. H.: The Relative Relief of Ohio. Geogr. Review, New York 1935.

\section{Verbandsnachrichten - Activité des sociétés}

\section{Planung - Hauptthema der Jahrestagung des Vereins schweizerischer Geographielehrer}

Am 15./16. November 1968 fand in Baden die Jahrestagung des Vereins Schweizerischer Geographielehrer statt. Stand schon 1967 das Thema «Landesplanung» im Mittelpunkt der Veranstaltungen der Tagung in Genf, so sollte 1968 die praktische Seite der Planung in den Vordergrund gerückt werden. Baden eignete sich als Studienobjekt sehr gut. Diese Gemeinde hat einen vollamtlichen Stadtplaner angestellt und steht jetzt gerade vor der Verwirklichung interessanter Projekte. Die allgemeine Verkehrssanierung hat bereits große Veränderungen im Stadtbild hervorgerufen. Durch eine sinnvolle Planung muß jetzt die Neugestaltung der Innenstadt so gelenkt werden, daß Baden zu einem modernen Regionalzentrum ausgebaut wird, ohne daß sein historisch gewachsenes Aussehen einfach von riesigen Geschäftshäusern erdrückt wird. Eine andere interessante Planungsaufgabe ist die Überbauung der bis 1961 selbständigen Gemeinde Dättwil. Darüber wurde 1966 ein Wettbewerb ausgeschrieben, dessen Resultate heute vorliegen. In den nächsten Jahren soll dieses Stück Agrarlandschaft von rund $1,3 \mathrm{~km}^{2}$ Fläche in einen Stadtteil umgewandelt werden, der Wohnraum für 6000 bis 8000 Einwohner und mindestens 2000 Arbeitsplätze umfassen wird. Diese beiden Hauptprobleme der Planungsbehörden von Baden wurden in gut dokumentierten Referaten von Stadtplaner Fritz Wagner, dipl. Arch. ETH, Vize-Stadtammann Josef Rieser, dipl. Arch. ETH, des Mitverfassers des erstprämiierten Pro- jekts A. Henz, dipl. Arch. ETH, des Stadtschreibers Dr. Victor Rickenbach, Präsident der Planungskommission von Baden und des Soziologen Dr. W. Vogt erläutert.

Eine Fahrt nach Dättwil zeigte deutlich den Gegensatz zwischen der heutigen und der anhand von Plänen und Modellen demonstrierten zukünftigen Situation dieses Gebietes. Solche Wandlungen des Landschaftsbildes müssen den Geographen interessieren. Sie eignen sich aber auch gut für die Behandlung im Unterricht an der Oberstufe unserer Mittelschulen. Wie gerade hier die Landesplanung eingebaut werden kann, erklärte auf anschauliche Weise Prof. Dr. Georges Grosjean in seinem vortrefflichen Referat über "Landesplanung als $\mathrm{Er}$ ziehungsaufgabe». Mit Dias und klaren Zahlenangaben untermauerte er seinen Appell an das Verantwortungsbewußtsein der heutigen Generation, dafür zu sorgen, daß nicht einfach im luftleeren Raum geplant wird. Die Summe aller Ortsplanungen ergibt keine vernünftige Landesplanung. Damit alles zusammenstimmt, muß man zuerst großräumig planen. Die katastrophale Auswirkung der Streubauweise auf die Infrastrukturkosten und die schädlichen Folgen einer falschen Planung zeigen die Notwendigkeit einer allgemeinen Aufklärung des ganzen Volkes über diese Probleme. Damit ist die Landesplanung wirklich eine Erziehungsaufgabe.

R. Wehrle 